\title{
Spectrum splitting photovoltaics: light trapping filtered concentrator for ultrahigh photovoltaic efficiency
}

\author{
Emily D. Kosten*a, Emily C. Warmann ${ }^{\mathrm{a}}$, John Lloyd ${ }^{\mathrm{a}}$, and Harry A. Atwater ${ }^{\mathrm{a}}$ \\ ${ }^{a}$ Thomas J. Watson Laboratories of Applied Physics, California Institute of Technology, \\ 1200 E. California Blvd., Pasadena, CA, USA 95555-0345
}

\begin{abstract}
While monolithic multijunction solar cell approaches have been quite successful, current and lattice matching requirements limit the maximum possible achievable efficiencies. Spectrum splitting, where light is optically distributed among subcells with differing bandgaps, avoids these constraints and offers a route to achieving higher efficiencies $(>50 \%)$. We investigate a spectrum splitting approach where concentrated sunlight is trapped in a textured dielectric slab and then selectively coupled into underlying solar cells of different bandgaps through omnidirectional filters. We develop a multipass optical model to find regimes of high optical efficiency based on parameters such as slab refractive index, number of subcells, and angle restriction of light escape from the slab. Based on these results and filter design considerations, we describe a specific design featuring a textured slab of $\mathrm{SiO}_{2}$ coated with angle restricting incoupling elements based on compound parabolic concentrators and three underlying multijunction junction solar cells, for a total of eight junctions with bandgaps ranging from $2.2 \mathrm{eV}$ to 0.7 . Using the multipass model in conjunction with modified detailed balance calculations, we find module efficiencies exceeding $50 \%$ are possible with an acceptance angle restricted to $20^{\circ}$ or less and concentrations of a few hundred suns with ideal omnidirectional filters. Finally as proof of concept, we design a full set of omnidirectional filters for this design. Based on alternating layers of $\mathrm{TiO}_{2}$ and $\mathrm{SiO}_{2}$, we achieve angle averaged reflectivity greater than $90 \%$ within the reflection band and angle averaged transmission of approximately $90 \%$ within the transmission band for the long pass filter, for nearly $48 \%$ receiver efficiency.
\end{abstract}

Keywords: spectrum splitting, multijunction solar cell, solar concentrator

\section{INTRODUCTION}

While the theoretical Landsberg limit for a solar cell is $86 \%$ current multijunction solar cells currently only achieve $37.9 \%$ under 1 sun. $^{1,2}$ Despite the significant benefits in greater numbers of junctions, current and lattice matching requirements limit the number of junctions in the highest quality cells, such that the current world record cell has only three junctions. ${ }^{2}$ Here, we pursue a different approach, by optically splitting the cell onto several multijunction cells that are optically and electrically in parallel, we reduce the losses from current and lattice matching requirements and allow for many more junctions. In this light trapping filtered concentrator approach, first proposed by Goetzberger, receiver efficiencies exceeding fifty percent may be attainable. ${ }^{3}$

\section{SUMMARY OF RESULTS}

The basic concept of the light trapping filtered concentrator approach is shown in figure 1. The entire receiver structure shown in the figure will be placed under a high concentration optic. Light then enters the receiver via an angle restricting optic made of an array of reflecting hexagonal compound parabolic concentrator structures. ${ }^{4,5}$ Spectral splitting occurs in the dielectric slab, where light is trapped by total internal reflection due to the randomizing texture of the slab and the reflectors underlying the angle restricting structure. Light then bounces randomly through the dielectric slab until it either escapes or enters one of the underlying filtered subcells. One of the key challenges of this spectral splitting approach is designing filters with omnidirectional performance for light incident from the glass slab.

High and Low Concentrator Systems for Solar Electric Applications VIII, edited by Adam P. Plesniak, Proc. of SPIE Vol. 8821, 882109 - (c) 2013 SPIE · CCC code: 0277-786X/13/\$18 · doi: 10.1117/12.2024275 


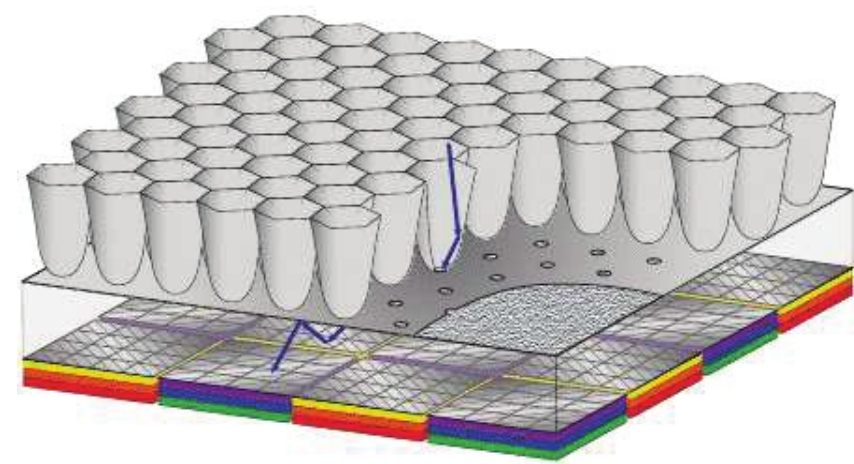

Figure 1. Schematic of the light trapping filtered concentrator receiver for use under an external concentrator. Light enters via a CPC array angle restrictor and is trapped by the angle restrictor reflector and total internal reflection in the textured dielectric slab. Light bounces within the textured dielectric slab until it escapes or enters one of the filtered multijunction solar cells arranged in an array below the slab.

Before filters can be designed, however, we must optimize the spectral bands for the underlying subcells. Based on a multipass optical model, we have determined that 2-4 underlying multijunction subcells should allow for high optical efficiency with feasible angle restriction and ideal filters. ${ }^{6}$ While two subcells allow for the highest optical efficiency, we are limited to six total junctions even with triple junction subcells. Once optical losses and realistic cell performance are included, six junctions are simply not enough to reach $50 \%$ efficiency. ${ }^{7}$. While in previous work we considered four underlying dual junctions, we achieved optical efficiencies somewhat lower than desirable with realistic filters. ${ }^{7}$ Therefore, our current design efforts focus on three subcells with two triple junctions and one dual junction. As shown in table 1, we first optimized three triple junction bandgaps for AM1.5D to find three current-matched triple junctions. These optimizations assume a 1\% external radiative efficiency (ERE), $90 \%$ of ideal current, and 500x concentration. ${ }^{7,8}$ However, the top bandgap was very high, such that it would be difficult to fabricate with high ERE. Therefore, we limited the top bandgap to $2.2 \mathrm{eV}$ and re-optimized. Finally, we found that eliminating the bottom junction had a minimal effect on the overall efficiency and avoided a very low bandgap that would be difficult to achieve with high ERE.

\begin{tabular}{|c|c|c|}
\hline $\begin{array}{c}\text { Overall Optimal Bandgaps } \\
\text { (3 Triple Junctions) }\end{array}$ & $\begin{array}{c}\text { Limited Top Bandgap } \\
\text { (3 Triple Junctions) }\end{array}$ & $\begin{array}{c}\text { Limited Top Bandgap } \\
\text { (2 Triple \& 1 Dual Junction) }\end{array}$ \\
\hline $2.57 \mathrm{eV}$ & $2.2 \mathrm{eV}$ & $2.2 \mathrm{eV}$ \\
$2.23 \mathrm{eV}$ & $1.78 \mathrm{eV}$ & $1.78 \mathrm{eV}$ \\
$1.99 \mathrm{eV}$ & $1.48 \mathrm{eV}$ & $1.48 \mathrm{eV}$ \\
\hline $1.63 \mathrm{eV}$ & $1.36 \mathrm{eV}$ & $1.37 \mathrm{eV}$ \\
\hline $1.36 \mathrm{eV}$ & $1.22 \mathrm{eV}$ & $1.23 \mathrm{eV}$ \\
\hline $1.12 \mathrm{eV}$ & $1.13 \mathrm{eV}$ & $1.15 \mathrm{eV}$ \\
\hline $0.93 \mathrm{eV}$ & $0.94 \mathrm{eV}$ & $0.93 \mathrm{eV}$ \\
\hline $0.72 \mathrm{eV}$ & $0.73 \mathrm{eV}$ & $0.70 \mathrm{eV}$ \\
\hline $0.39 \mathrm{eV}$ & $0.52 \mathrm{eV}$ & --------- \\
\hline Efficiency: 55.7\% & Efficiency: 54.1\% & Efficiency: 53.5\% \\
$(500 \mathrm{x} 1 \% \mathrm{ERE})$ & $(500 \mathrm{x} 1 \% \mathrm{ERE})$ & $1 \% \mathrm{ERE})$ \\
\hline
\end{tabular}

Table 1. Optimized bandgaps for multijunction subcells. The left column is the overall optimum for AM 1.5D. The center column limits the top bandgap to $2.2 \mathrm{eV}$, and the rightmost column utilizes only a dual junction in the lowest energy subcell. 
Based on these spectral bands, we then designed a set of three filters based on $\mathrm{TiO}_{2}$ and $\mathrm{SiO}_{2}{ }^{9}$ The large index contrast increases the omnidirectional reflectivity of the structures. For each of these designs, we started with a chirped dielectric structure that was then optimized in accordance with reflection and transmission targets at various angles. We then input the angle-averaged filter performance into our multipass optical model. We define a tracking accuracy as the product of the external concentration and $1 / \sin ^{2} \theta$, where $\theta$ is the acceptance angle of the angle restrictor. Assuming 10,000x tracking accuracy, 20\% parasitic absorption loss for light that enters a lower bandgap cell, $90 \%$ of ideal Jsc and $1 \%$ cell ERE, we find receiver efficiencies approaching $48 \%$ are feasible. With higher quality (3\% ERE) cells and $30,000 \mathrm{x}$ tracking accuracy, receiver efficiencies greater than 50\% may be achieved with these initial filter designs.

\section{CONCLUSIONS}

We have investigated the light trapping filtered concentrator with three underlying multijunction subcells. We have optimized these subcells to consist of two triple junctions and one dual junction, bearing in mind reasonable materials choices. We have also developed a multipass optical model that we use in conjunction with an initial design of three $\mathrm{TiO}_{2} / \mathrm{SiO}_{2}$ multilayer omnidirectional filters. Using this approach, we determine that for realistic cells and tracking accuracies receiver efficiencies approaching $48 \%$ are achievable.

\section{REFERENCES}

[1] Green, M.A. [Third Generation Photovoltaics], Springer, New York, (2006).

[2] Green, M.A. et al. "Solar cell efficiency tables (version 42)," Prog. in Photovoltaics 21, 827-837 (2013).

[3] Goetzberger A. et al., "Light trapping, a new approach to spectrum splitting," Solar Energy Materials and Solar Cells, 92, 1570-1578 (2008).

[4] Welford W.T. and Winston R. [High Collection Non-Imaging Optics]. Academic Press, San Diego, (1989).

[5] Kosten E.D. et al., "Highly efficient GaAs solar cells by limiting light emission angle," Light: Science \& Applications, 2, e45, (2013).

[6] Kosten, E.D., Warmann, E.C., Lloyd, J. and Atwater, H.A. "Spectrum Splitting Photovoltaics: Light Trapping Filtered Concentrator for Ultrahigh Photovoltaic Efficiency," Proc. $39^{\text {th }}$ IEEE PVSC, (2013).

[7] Warmann, E.C., et al. "Spectrum splitting photovoltaics: proposed band gaps and materials to achieve $>50 \%$ system efficiency," Proc. $39^{\text {th }}$ IEEE PVSC, (2013).

[8] Green, M.A. "Radiative efficiency of state-of-the-art photovoltaic cells," Progress in Photovoltaics: Research and Applications, 20, 472-476 (2011).

[9] Larouche S. and Martinu L., "OpenFilters: open-source software for the design, optimization, and synthesis of optical filters," Applied Optics, 47, 219-230 (2008). 